\title{
Is the Care Transitions Measure Associated with Readmission Risk? Analysis from a Single Academic Center
}

\author{
Jennifer N. Goldstein, M.D., M.Sc. ${ }^{1,2}$, LeRoi S. Hicks, M.D., M.P.H. ${ }^{1}$, Paul Kolm, Ph.D. ${ }^{2}$, \\ William S. Weintraub, M.D. ${ }^{2,3}$, and Daniel J. Elliott, M.D., M.S.C.E. ${ }^{2,4}$
}

'Department of Medicine, Christiana Care Health System, Newark, DE, USA; ${ }^{T}$ The Value Institute, Christiana Care Health System, Newark, DE, USA; ${ }^{3}$ Division of Cardiology, Christiana Care Health System, Newark, DE, USA; ${ }^{4}$ Christiana Care Quality Partners, Christiana Care Health System, Newark, $D E$, USA.

BACKGROUND: It is widely hypothesized that improvement in transitions of care will reduce unplanned hospital readmissions. However, the association between the Care Transitions Measure, the national quality metric for transitions of care and readmission risk, has not been established.

OBJECTIVE: We aimed to determine the association between the Care Transition Measure and readmission.

DESIGN: This was a single-center, prospective cohort study.

PARTICIPANTS: Convenience sample of 2,963 patients enrolled in the "Bridging the Divides" program, a longitudinal care management program for patients with coronary revascularization, from 2013 to 2014. Of these, 1594 (54\%) patients completed a post-discharge Care Transition Measure questionnaire.

INTERVENTION: Care Transition Measure scores were collected by trained research staff blinded to study hypothesis, by telephone, within 30 days of discharge. Higher Care Transition Measure scores reflect a higher quality transition of care.

MAIN MEASURES: 30-day readmission was measured. KEY RESULTS: Of the 1594 patients that completed the Care Transition Measure survey, 1216 (76\%) received percutaneous coronary intervention and $378(24 \%)$ received coronary artery bypass grafting. Mean Care Transition Measure scores were significantly lower among patients who had a prior admission (77.2 vs. $82.1, p<0.001)$ and those with $\geq 5$ comorbidities ( 77 vs. 82.6 vs. 81.6 , $p<0.001)$. Mean scores were significantly lower among patients who were readmitted within the percutaneous coronary intervention subgroup (73 vs. $80.9, p<0.001$ ) and the total study population (74.6 vs. $81.1, p<0.001$ ) compared to those who were not readmitted. This was not the case in the coronary artery bypass grafting subgroup (78.5 vs. 81.7, $p=0.29$ ). After multivariable adjustment, every ten-point increase in the Care Transition Measure score was associated with a $14 \%$ reduction in readmission risk (adjusted odds ratio 0.86, $95 \%$ CI 0.78-0.95).

CONCLUSIONS: The Care Transition Measure is strongly associated with readmissions, which strengthens its validity. However, its association with patient variables

Prior Presentations This work was presented at the 2015 Mid-Atlantic and National Society for General Internal Medicine Conferences.

Received June 9, 2015

Revised December 4, 2015

Accepted January 21, 2016

Published online February 11, 2016 linked with readmission and its inconsistent association with readmission across clinical groups raises concerns that scores may be influenced by patient characteristics.

KEY WORDS: patient satisfaction; reimbursement; care transitions; cardiovascular disease.

J Gen Intern Med 31(7):732-8

DOI: $10.1007 / \mathrm{s} 11606-016-3610-9$

(c) Society of General Internal Medicine 2016

\section{BACKGROUND}

The transition from hospital to home is a vulnerable time for patients and their families. Poor care coordination during this time increases the risk for potentially avoidable hospital readmissions. ${ }^{1}$ Such readmissions place a tremendous burden on patients and families and cost the US health system approximately $\$ 12$ billion annually. ${ }^{2}$ Unlike other risk factors for readmission, such as race, sex and age and prior hospitalization, ${ }^{3}$ poor transitions of care can be remediated with improved care management. ${ }^{4,5}$ As a result, they have become a target for intervention and improvement. On a national level, over $\$ 300$ million has been invested in transitions of care programs by the Centers for Medicare and Medicaid with the explicit goal of reducing readmissions. ${ }^{6}$ In addition, sizable investments in transitions of care interventions have been made by public and private grant-making organizations and hospital systems. ${ }^{4,7-11}$

The introduction of performance-based hospital reimbursement adds financial incentives to reduce readmissions and improve transitions of care. Under the Hospital Readmissions Reduction Program, Medicare incurs financial penalties on hospitals with higher than expected rates of readmissions. Although the program only applies to patients with a primary diagnosis of congestive heart failure, acute myocardial infarction, or pneumonia, the Medicare Payment Advisory Commission has recommended inclusion of patients with chronic obstructive pulmonary disease, coronary artery bypass grafting (CABG), and percutaneous coronary intervention (PCI) in $2015 .^{12}$ As hospitals are held financially accountable for a greater proportion of readmissions, investments in programs to reduce readmissions, such as transition of care programs, hold greater potential value.

Additionally, Medicare will now reimburse hospitals differentially based on patient perception of the quality of transitions 
of care. Under the Value-Based Purchasing Program, hospitals are reimbursed based on patient responses to the Hospital Consumer Assessment of Healthcare Providers and Systems (HCAHPS) patient satisfaction survey. As of 2013, a quality metric for transitions of care, called the Care Transitions Measure (CTM-3) was added to the HCAHPS survey (See Text Box). Therefore, hospitals are now reimbursed differentially based on their performance on the CTM-3.

\footnotetext{
The Care Transitions Measure (CTM-3)

- The hospital staff took my preferences and those of my family or caregiver into account in deciding what my health care needs would be when I left the hospital.

- When I left the hospital I had a good understanding of the things I was responsible for in managing my health.

- When I left the hospital I clearly understood the purpose for taking each of my medications.
}

The addition of the CTM-3 to HCAHPS has raised questions about the validity of the metric itself and its role in the evaluation of transitions of care. The CTM-3 is a threequestion instrument that evaluates three key domains related to transitions of care: whether patient and family's preferences were accounted for in the care plan, whether patients understood their role in self-management after discharge, and whether appropriate medication education was provided. Higher scores on the CTM-3 reflect higher quality transitions of care. ${ }^{13}$ Although low CTM-3 scores have been associated with emergency room revisits, ${ }^{14}$ they have not been associated with inpatient readmissions that are subjected to Hospital Readmissions Reduction Program penalties. Additionally, in a previous validation study, the study population was less than 300 patients and almost entirely Caucasian. ${ }^{14}$

Given the large investments that have been made by CMS and hospital systems on transitions of care programs with the hope that they will reduce readmissions, it is important to know whether the CTM-3, the national quality metric for transitions of care, is associated with readmissions. Additionally, given its recent incorporation into the HCAHPS survey, it is important to understand how the metric performs in a larger, more representative patient population and how that may impact hospital reimbursements. Therefore, the primary objective of this analysis is to determine the association between the CTM-3 and readmissions, and to validate the CTM-3 within a larger and more diverse patient population.

\section{METHODS}

Design. Data were collected as part of a quality improvement program for patients who received coronary revascularization at the Christiana Care Health System, the largest regional academic health system in Delaware. The program was called "Bridging the Divides" ("Bridges") and was sponsored by the Center for Medicare and Medicaid Innovation. The program was approved by the Christiana Care Institutional Review Board. Because the Bridges program was a quality improvement initiative, patients were not required to provide informed consent. However, approval to publish results of the study was obtained.

Participants. All patients undergoing coronary revascularization with $\mathrm{PCI}$ or CABG at the Christiana Care Health System between April 2013 and November 2014 $(n=2,963)$ were enrolled in the Bridges program. Of these, 1668 completed the CTM-3 survey. A small percentage, $4.4 \%$ $(74 / 1,668)$, had evidence of significant coronary artery disease on cardiac catheterization but did not undergo revascularization. These patients were excluded from the analysis because their readmission risk was potentially altered because intervention was withheld. This left 1,594 patients for analysis.

All patients underwent a readmission risk assessment at enrollment, specific to the Bridges program, which was based on clinical, demographic, and prior health care utilization characteristics. Patients with high readmission risk scores received medication education from a clinical pharmacist and their bedside nurse, and biweekly phone calls during the first 2 weeks post-discharge, followed by regularly scheduled calls from a care management nurse for a total of 1 year. Patients with mid-range to low readmission risk scores received at least two post-discharge phone calls over the first month and regular follow-up calls from a social worker or health coach for a total of 1 year. The CTM-3 survey was administered to all patients as part of their telephone assessment within the first 30 days of discharge (median 2 days, inter-quartile range 1-4). Over the course of the program, if no response was obtained after three consecutive phone calls, patients were discontinued from the Bridges Program.

Main Measures. The primary predictor variable was the CTM-3 score, obtained from the Bridges study database. The primary outcome was all-cause, 30-day non-elective readmission abstracted from the electronic medical record. Demographic data such as race, sex, discharge location (home, home with health care services/ facility), primary insurance (Medicare, Medicaid, private, self-pay/other), age, hospitalization in the 6 months prior to admission (yes/no), and comorbidity count also were abstracted from the electronic medical record. Patients who had Medicare and Medicaid were categorized as Medicare patients in our analysis. Comorbidity counts were calculated by summing the 29 Elixhauser diagnosis-related group categories pertinent to each patient. ${ }^{15}$

Analyses. We utilized the Chi-square test to examine differences between the PCI and CABG populations and patients that did and did not complete the CTM-3 survey. CTM-3 was scored and converted to a 100-point scale as recommended by 
the National Quality Forum, ${ }^{16}$ and analyzed as a continuous variable. ANOVA analysis was used to determine the relationship between mean CTM-3 scores and variables associated with readmissions in prior studies, including race, gender, age, comorbidity count, prior hospital utilization, ${ }^{3}$ insurance status and discharge location. Three separate analyses were performed for those with PCI, CABG, and the combined population of PCI \& CABG. A multivariable model was developed that adjusted for sociodemographic and clinical characteristics including procedure (CABG or $\mathrm{PCI})$.

To determine whether CTM-3 score independently predicted 30-day non-elective readmission, given the exploratory nature of the study, we included all variables that had been used in the bivariate ANOVA analyses in the multivariate model. Log pseudo-likelihood ratios of multivariate models with and without CTM-3 were compared to determine the statistical significance of observed differences and whether addition of the CTM-3 measure improved the model's predictive capacity.

\section{RESULTS}

\section{Patient Characteristics (Table 1)}

Of 2963 total patients, 1668 (56 \%) completed CTM-3 surveys, and 1594 (54\%) received coronary revascularization

Table 1. Characteristics of Patients with and without CTM-3 Scores"

\begin{tabular}{|c|c|c|c|}
\hline Variable & $\begin{array}{l}\text { CTM-3 } \\
N(\%)\end{array}$ & $\begin{array}{l}\text { No CTM-3 } \\
N(\%)\end{array}$ & $p$ value \\
\hline \multicolumn{3}{|l|}{ Race } & \multirow[t]{4}{*}{0.41} \\
\hline Caucasian & $1311(82.2)$ & $1149(83.9)^{\dagger}$ & \\
\hline African American & $197(12.4)$ & $158(11.5)$ & \\
\hline Other & $86(5.3)$ & $62(4.5)$ & \\
\hline \multicolumn{3}{|l|}{ Sex } & \multirow[t]{3}{*}{0.60} \\
\hline Male & $1117(70.1)$ & $947(69.2)$ & \\
\hline Female & 477 (29.9) & $422(30.8)$ & \\
\hline \multicolumn{3}{|l|}{ Discharge location } & \multirow[t]{4}{*}{0.59} \\
\hline Home & $1099(69.0)$ & $890(68.0)$ & \\
\hline Home with services / & $494(31.0)$ & $418(32.0)$ & \\
\hline \multicolumn{3}{|l|}{ Facility } & \\
\hline Insurance & & & \multirow[t]{5}{*}{0.02} \\
\hline Medicare & $816(51.3)$ & $780(57.0)$ & \\
\hline Medicaid & $136(8.5)$ & $102(7.5)$ & \\
\hline Private & $584(36.7)$ & $442(32.3)$ & \\
\hline Self- pay / other & $58(3.6)$ & $45(3.3)$ & \\
\hline \multicolumn{3}{|l|}{ Age } & \multirow[t]{4}{*}{0.09} \\
\hline$>65$ & $764(47.9)$ & $701(51.2)$ & \\
\hline $50-65$ & $670(42.0)$ & $556(40.6)$ & \\
\hline$<50$ & $160(10.0)$ & $112(8.2)$ & \\
\hline \multicolumn{3}{|l|}{ Hospitalization in previous } & \multirow[t]{4}{*}{$<0.001$} \\
\hline \multicolumn{3}{|l|}{6 months } & \\
\hline Yes & $475(29.8)$ & $573(41.9)$ & \\
\hline No & $1119(70.2)$ & $796(58.1)$ & \\
\hline \multicolumn{3}{|l|}{ Elixhauser count } & \multirow[t]{4}{*}{$<0.001$} \\
\hline $0-2$ & $674(42.3)$ & $430(31.4)$ & \\
\hline 3,4 & $436(27.4)$ & $365(26.7)$ & \\
\hline$\geq 5$ & $484(30.4)$ & $574(41.9)$ & \\
\hline \multicolumn{3}{|l|}{30 Day readmission } & \multirow[t]{3}{*}{$<0.001$} \\
\hline Yes & $106(6.6)$ & $153(11.2)$ & \\
\hline No & $1488(93.4)$ & $1216(88.8)$ & \\
\hline
\end{tabular}

*Totals may not sum to 2,963 because of missing values

+ Column percentages may not sum to $100 \%$ because of rounding error and were included in the analysis. Of patients with PCI, 1216/ 2292 (53 \%) completed CTM-3 surveys, while 378/671 (56\%) of patients with CABG completed the survey. Patients who did not complete the CTM-3 survey were more likely to have Medicare as the primary form of insurance $(57.0 \% \mathrm{vs}$. $51.3 \%, p=0.019)$, five or more comorbidities ( $41.9 \%$ vs. $30.4 \%, p<0.001)$ and a previous admission (41.9\% vs. $29.8 \%, p<0.001)$ than those who completed the survey. The risk for 30-day all-cause readmission was $6.6 \%$ for those who completed the CTM-3 versus $11.2 \%$ among those who did not $(p<0.001)$.

Among the 1594 patients who completed the CTM-3, 197 (12.4\%) were African American, 1,117 (70.1\%) were male, $1434(89.9 \%)$ were over the age of 50, $484(30.4 \%)$ had five or more comorbidities, $136(8.5 \%)$ had Medicaid, and 816 $(51.3 \%)$ had Medicare as their primary form of insurance. CABG patients were more likely than PCI patients to have five or more comorbidities $(44.2 \%$ vs. $26.2 \%, p<0.001)$, and were more likely to be discharged with home health care or to a facility ( $90.0 \%$ vs. $12.4 \%, p<0.001)$, to have been hospitalized in the previous 6 months $(47.1 \%$ vs. $24.4 \% p<0.001)$, and be male ( $77 \%$ vs. $68 \%, p<0.001$ ), but did not differ statistically in other socioeconomic or demographic variables or readmission risk ( $8.2 \%$ vs. $6.2 \%, p=0.166)$.

\section{Patient Variables Associated with CTM-3 Scores: (Table 2)}

Table 2 shows variables associated with CTM-3 scores in the PCI, CABG and combined populations. Within all groups, patients with five or greater comorbidities reported lower mean CTM-3 scores than those with fewer than five comorbidities. Within the PCI population, patients with a prior admission reported a 6.3-point lower mean CTM-3 score compared to those without a prior hospitalization; within the combined population, patients with prior hospitalization reported a 4.8-point lower mean $\mathrm{CTM}^{-} 3$ score than those without a prior hospitalization (both $p<0.001$ ).

\section{CTM-3 and Readmission (Tables 2 and 3)}

Within the PCI and the total population, lower mean CTM3 scores were significantly associated with higher risk of readmission. There was no statistically significant association between mean CTM-3 scores and readmission within the CABG group (Table 2). Within the total population of patients, after multivariable adjustment of patient's clinical and sociodemographic characteristics, we found that for every ten-point increase in CTM-3 score, patients had $14 \%$ lower odds of readmission to the hospital (Table 3). A ten-point increase in CTM-3 translates to a change in a response of one out of three CTM-3 questions to the next highest response category ${ }^{16}$; for example, a change from a response of "Agree" for all three questions to a response of "Strongly Agree" for one question 
Table 2. Bivariate Analysis of Mean CTM-3 Scores* $(n=1594)$

\begin{tabular}{|c|c|c|c|c|c|c|c|c|c|}
\hline \multirow[b]{2}{*}{ Variable } & \multicolumn{3}{|c|}{ CABG } & \multicolumn{3}{|l|}{ PCI } & \multicolumn{3}{|c|}{ PCI \& CABG } \\
\hline & $n$ & Mean \pm SD & $p$ value & $n$ & Mean \pm SD & $p$ value & $n$ & Mean \pm SD & $p$ value \\
\hline Race & & & 0.86 & & & 0.32 & & & 0.31 \\
\hline Caucasian & 312 & $81.7 \pm 16.8$ & & 999 & $80.6 \pm 17.8$ & & 1311 & $80.9 \pm 17.6$ & \\
\hline African American & 41 & $80.6 \pm 15.5$ & & 156 & $79.9 \pm 16.4$ & & 197 & $80.03 \pm 16.2$ & \\
\hline Other & 25 & $80.2 \pm 13.2$ & & 61 & $77.2 \pm 17.4$ & & 86 & $78.1 \pm 16.3$ & \\
\hline Sex & & & 0.06 & & & 0.98 & & & 0.38 \\
\hline Male & 291 & $82.3 \pm 16.0$ & & 826 & $80.4 \pm 17.1$ & & 1117 & $80.9 \pm 16.9$ & \\
\hline Female & 87 & $78.5 \pm 17.3$ & & 390 & $80.4 \pm 18.6$ & & 477 & $80.04 \pm 18.4$ & \\
\hline Discharge location & & & 0.74 & & & 0.05 & & & 0.55 \\
\hline Home & 34 & $82.3 \pm 15.5$ & & 1065 & $80.8 \pm 17.5$ & & 1099 & $80.8 \pm 17.4$ & \\
\hline Home with services / Facility & 344 & $81.4 \pm 16.5$ & & 150 & $77.7 \pm 17.6$ & & 494 & $80.3 \pm 16.9$ & \\
\hline Insurance & & & 0.04 & & & 0.23 & & & 0.10 \\
\hline Medicare & 198 & $81.0 \pm 17.1$ & & 618 & $80.0 \pm 16.6$ & & 816 & $80.3 \pm 16.8$ & \\
\hline Medicaid & 35 & $75.1 \pm 12.5$ & & 101 & $79.8 \pm 18.9$ & & 136 & $78.6 \pm 17.5$ & \\
\hline Private & 126 & $83.8 \pm 15.8$ & & 458 & $81.3 \pm 18.4$ & & 584 & $81.9 \pm 17.9$ & \\
\hline Self- pay / other & 19 & $83.0 \pm 16.7$ & & 39 & $75.8 \pm 18.9$ & & 58 & $78.2 \pm 18.4$ & \\
\hline Age (years) & & & 0.12 & & & 0.60 & & & 0.99 \\
\hline$>65$ & 196 & $81.5 \pm 16.1$ & & 568 & $80.2 \pm 17.5$ & & 764 & $80.6 \pm 17.1$ & \\
\hline $50-65$ & 152 & $82.5 \pm 15.8$ & & 518 & $80.2 \pm 17.9$ & & 670 & $80.7 \pm 17.4$ & \\
\hline$<50$ & 30 & $75.7 \pm 20.3$ & & 130 & $81.8 \pm 17.0$ & & 160 & $80.7 \pm 17.8$ & \\
\hline Hospitalization in previous 6 months & & & 0.05 & & & $<0.001$ & & & $<0.001$ \\
\hline Yes & 178 & $79.7 \pm 17.1$ & & 297 & $75.6 \pm 18.8$ & & 475 & $77.2 \pm 18.2$ & \\
\hline No & 200 & $83.0 \pm 15.7$ & & 919 & $81.9 \pm 16.9$ & & 1119 & $82.1 \pm 16.7$ & \\
\hline Elixhauser count & & & 0.05 & & & $<0.001$ & & & $<0.001$ \\
\hline $0-2$ & 92 & $83.3 \pm 15.6$ & & 582 & $82.5 \pm 17.3$ & & 674 & $82.6 \pm 17.1$ & \\
\hline $3-4$ & 119 & $83.3 \pm 16.4$ & & 317 & $80.9 \pm 17.5$ & & 436 & $81.6 \pm 17.2$ & \\
\hline$\geq 5$ & 167 & $79.1 \pm 16.7$ & & 317 & $75.8 \pm 17.5$ & & 484 & $77.0 \pm 17.2$ & \\
\hline 30-day readmission & & & 0.29 & & & $<0.001$ & & & $<0.001$ \\
\hline Yes & 31 & $78.5 \pm 14.6$ & & 75 & $73 \pm 17.2$ & & 106 & $74.6 \pm 17.3$ & \\
\hline No & 347 & $81.7 \pm 16.5$ & & 1141 & $80.9 \pm 17.5$ & & 1488 & $81.1 \pm 16.6$ & \\
\hline
\end{tabular}

*Three separate ANOVA analyses were performed

Table 3. Logistic Regression with 30-day All-Cause Readmission as Outcome; $N=1593$

\begin{tabular}{|c|c|c|}
\hline Variable & Odds ratio & $95 \%$ confidence interval \\
\hline \multicolumn{3}{|l|}{ Race } \\
\hline White & 1.00 & - \\
\hline Black & 1.22 & $0.69-2.17$ \\
\hline Other & 1.01 & $0.42-2.44$ \\
\hline \multicolumn{3}{|l|}{ Sex } \\
\hline Female & 1.00 & - \\
\hline Male & 1.11 & $0.70-1.77$ \\
\hline \multicolumn{3}{|l|}{ Discharge disposition } \\
\hline Home & 1.00 & - \\
\hline Home w/ svc / Facility & 1.68 & $0.70-1.77$ \\
\hline \multicolumn{3}{|l|}{ Insurance } \\
\hline Medicare & 1.00 & - \\
\hline Medicaid & 0.88 & $0.41-1.91$ \\
\hline Private & 0.50 & $0.27-0.91$ \\
\hline Self-pay / other & 0.80 & $0.26-2.41$ \\
\hline \multicolumn{3}{|l|}{ Age category } \\
\hline$>65$ & 1.00 & - \\
\hline $50-65$ & 0.80 & $0.39-1.66$ \\
\hline$<50$ & 0.58 & $0.26-1.31$ \\
\hline \multicolumn{3}{|l|}{ Elixhauser Count } \\
\hline $0-2$ & 1.00 & - \\
\hline 3,4 & 1.21 & $0.64-2.31$ \\
\hline$\geq 5$ & 2.89 & $1.67-4.9$ \\
\hline \multicolumn{3}{|c|}{ Hospitalization in previous 6 months } \\
\hline No & 1.00 & - \\
\hline Yes & 0.98 & $0.62-1.55$ \\
\hline \multicolumn{3}{|l|}{ Procedure } \\
\hline PCI & 1.00 & $0.39-1.56$ \\
\hline CABG & 0.78 & \\
\hline CTM-3 score $^{\dagger}$ & 0.86 & $0.78-0.95$ \\
\hline
\end{tabular}

*Odds ratios obtained utilizing multivariable logistic regression controlling for each factor presented in table and condition of interest. Pseudo R : 0.068; Wald Chi ${ }^{2}: 50.91$

+ Odds ratio calculated per ten-point increase in CTM-3 score and "Agree" for two questions. The $14 \%$ risk reduction that we found is comparable to the differential risk of hospital readmission for patients less than 55 years of age compared to those between the ages of 85 and 89 years. $^{3}$

When the models with and without the CTM-3 measure were compared, the multivariate model that included the CTM-3 variable had a stronger predictive capacity for readmissions than did the model without CTM-3 $(p=0.005)$. However, addition of the CTM-3 did not change the directionality of the odds ratios or predictive significance of any other variables in the model.

\section{DISCUSSION}

\section{CTM- -3 and Readmission}

Our findings at a single, large academic medical center suggest that hospitals that can increase their CTM-3 scores may be able to reduce their readmission rates. Our data supports the validity of the CTM-3 metric and its potential utility as a target for transitions of care programs. However, our analysis raised questions regarding the durability of the association between CTM-3 and readmissions across a clinically diverse patient population. Although we found a significant relationship between the CTM-3 and readmission within the PCI and total populations, we did not find a statistically significant relationship in the more debilitated CABG population. This 
challenges whether the CTM-3 adequately captures the posttransition experience for certain groups of patients. Patients with PCI are often readmitted for different reasons ${ }^{17}$ than patients with $\mathrm{CABG},{ }^{18}$ and many readmissions are not easily attributable to a single cause. ${ }^{1,19}$ Therefore, even if the three domains of the CTM-3 were addressed equally for patients with $\mathrm{PCI}$ and $\mathrm{CABG}$, their post transition experiences and ultimate reasons for readmission could vary greatly. Although our study generally supports the association between the CTM-3 score and readmission, it should be interpreted with caution, and the association should be validated on a large scale, at the hospital level in future studies.

\section{Implications for CTM-3 in Quality Improvement and Hospital Reimbursements}

We found that the CTM-3 was associated with non-modifiable patient characteristics previously associated with readmissions, particularly whether the patient had had a prior admission and whether they had a high number of comorbidities. This raises the question of whether, in addition to its association with known patient variables associated with readmission, the CTM-3 also accounts for unmeasured, fixed patient characteristics associated with readmission. It also questions whether the CTM-3 itself is fixed or whether it is modifiable.

To our knowledge, there is only one randomized controlled trial that examined the modifiability of CTM-3 scores; it demonstrated that CTM-3 scores could be significantly improved through an intensive care transitions program that provided primary care coordination, nursing and pharmacy counseling to a clinically diverse, low income population. However, the amount of improvement (i.e., change in mean CTM-3 scores) was not reported. ${ }^{20}$ An additional study suggested that the CTM-3 was modifiable, but not consistently. ${ }^{14}$

The association between the CTM-3 and fixed patient characteristics has potentially important implications for hospital reimbursement Under the Value Based Purchasing Program. Under the Value Based Purchasing program, Medicare adjusts payments to hospitals based in part on their performance on CTM-3 compared to all hospitals, or on their improvement in CTM-3 performance compared to a prior time period. ${ }^{21}$ If sicker patients have lower CTM-3 scores, it is plausible that hospitals could be inadvertently be penalized for caring for sicker populations. Furthermore, the ability of an institution to improve their score will rely on whether the CTM-3 can be improved, which has not been confirmed.

\section{Implications for Use of CTM-3 as a Readmission Risk Predictor}

Another important implication of our findings concerns the potential utility of CTM-3 in future risk prediction calculations. CTM-3 scores were independently associated with readmission and our predictive model was improved by the addition of the CTM-3 score. Thus, future studies should evaluate the utility of the CTM-3 as a readmission risk predictor in the peri- or post-discharge setting. Readmission risk prediction models are not generally used in the outpatient setting. ${ }^{22}$ However, with many hospital level and national initiatives focused on transitions of care, the development of a post-discharge risk assessment instrument for readmission may prove useful in the ongoing evaluation of patient readmission risk once they enter into the ambulatory setting. Such an assessment could trigger more intensive follow-up and care coordination after discharge, such as a nursing call or visiting nurse consultation.

Importantly, the utility of CTM-3 in risk prediction models may be limited by its potentially inconsistent association with readmission across patient populations, as mentioned above. Therefore, future studies should examine the association between CTM-3 and readmissions across a wide spectrum of patients and disease processes.

\section{Limitations}

This is a single center study, which is the primary limitation of our analysis. Our CTM-3 response rate was $54 \%$, and nonresponders were significantly different from our responders in several important respects. Therefore, our results are not generalizable. However, future analyses of the CTM-3 as part of the HCAHPS survey will encounter an even lower response rate of approximately $30 \% .{ }^{23}$ Response bias is a common and important limitation of patient satisfaction surveys. Prior research has illustrated that patients who are more satisfied with their care are more likely to respond to patient satisfaction surveys. ${ }^{24}$ This can lead to inflated satisfaction scores. ${ }^{24}$ In the context of our study, this implies that the mean CTM-3 scores of our non-responders were potentially lower, on average, than those of our responders. We also found an association between non-response and hospitalization, consistent with to prior research. ${ }^{25}$ In this context, it is plausible that our non-responders would have had lower CTM-3 scores and higher readmission risk. As a result, the association that we found between low CTM-3 scores and readmission risk among our responders could be an underestimate.

The majority of our sample was male, which does not reflect the true distribution of ischemic heart disease, but may reflect lower rates of invasive cardiovascular interventions on women. ${ }^{26,27}$ However, there were no statistically significant differences observed in CTM-3 scores or risk of readmission related to patient sex in our analysis. We did not account for readmissions to other hospital systems, which could skew the association of CTM-3 and readmission in either direction. However, Delaware residents accounted for $73 \%$ of our study population, and Christiana Care Health System accounts for the care of $80-90 \%$ of New Castle County, Delaware residents, so we likely captured the majority of readmissions and drew from a diverse and representative sample of patients. Future studies of national administrative data sets and HCAHPS records could address this limitation. Our analysis only looked at patients who underwent CABG 
and PCI. However, the clinical differences between the CABG and PCI populations offered two distinct patient samples in which to evaluate CTM-3 scores. It is important to note that our patient sample was enrolled in a care management program that sought to mitigate potential issues surrounding transitions of care, and responded to poor ratings on CTM-3 questions with care management interventions. Therefore, readmission risk among those with the lowest CTM-3 scores may have been lower than would have occurred without the intervention. If so, the observed relationship between CTM-3 and readmissions may again underestimate the relationship between CTM-3 and hospital readmission. Lastly, our analysis was observational, so causality cannot be inferred from our results.

\section{CONCLUSIONS}

To our knowledge, ours is the first large-scale analysis to reveal an association between CTM-3 and hospital readmissions. We found that a modest improvement in CTM-3 scores was independently associated with a large reduction in readmission risk. This validates the CTM-3 metric and supports its potential value in the assessment of post-discharge readmission risk. However, questions still remain regarding the durability of this association across patient populations and whether the CTM-3 score is modifiable. Given that CTM-3 is now a national quality metric, tied to hospital reimbursements, these remaining questions are particularly significant and lead us to question whether the CTM3 was implemented prematurely, before it was completely understood. Large-scale studies could improve our understanding of the ultimate utility of the CTM-3 and could help to clarify our expectations of this quality metric by which all US hospital systems will ultimately be measured.

\section{Acknowledgements:}

JNG had full access to all of the data in the study and takes responsibility for the integrity of the data and the accuracy of the data analysis.

Contributors: The authors would like to thank J. Sanford Schwartz MD MBA, Leon Hess Professor of Internal Medicine, University of Pennsylvania, and Allan S. Detsky, MD PhD, Professor of Medicine, Department of Medicine, Mount Sinai Hospital, for their thoughtful review of the manuscript.

Corresponding Author: Jennifer N. Goldstein, M.D., M.Sc.; Department of Medicine, Christiana Care Health System, 4755 OgletownStanton Road, Ammon Education Building, Suite 2E7O, Newark, DE 19713, USA (e-mail:jgoldstein@christianacare.org).

\section{Compliance with ethical standards:}

Funders: The project described was supported by Grant Number 1C1CMS331027 from the Department of Health and Human Services, Centers for Medicare \& Medicaid Services. The content of this presentation are solely the responsibility of the authors and have not been approved by the Department of Health and Human Services, Centers for Medicare \& Medicaid Services.

Dr. Goldstein and Dr. Hicks are supported by and Institutional Development Award (IDeA) from the National Institute of General Medical
Sciences of the National Institutes of Health under grant number U54GM104941 (PI: Binder-Macleod).

The above funding organizations had no role in the design and conduct of the study; collection, management, analysis, and interpretation of the data; and preparation, review, or approval of the manuscript; and decision to submit the manuscript for publication.

Conflict of Interest: The authors declare that they do not have a conflict of interest.

\section{REFERENCES}

1. Kangovi S, Barg FK, Carter T, et al. Challenges faced by patients with low socioeconomic status during the post-hospital transition. J Gen Intern Med. 2014;29(2):283-9.

2. Burton R. Health policy brief: care transitions. Health Affairs, 2012. http://healthaffairs.org/healthpolicybriefs/brief_pdfs/healthpolicybrief 76.pdf. Accessed 1/4/2016

3. Jencks SF, Williams MV, Coleman EA. Rehospitalizations among patients in the Medicare fee-for-service program. N Engl J Med. 2009;360(14):1418-28.

4. Naylor MD, Brooten D, Campbell R, et al. Comprehensive discharge planning and home follow-up of hospitalized elders: a randomized clinical trial. JAMA. 1999;281(7):613-20.

5. Kangovi S, Mitra N, Grande D, et al. Patient-centered community health worker intervention to improve posthospital outcomes: a randomized clinical trial. JAMA Intern Med. 2014; 174(4):535-43.

6. Centers for Medicare and Medicaid Services. Community-based care transitions program. http://innovation.cms.gov/initiatives/CCTP/?itemid=CMS1239313. Accessed 1/4/2016.

7. Verhaegh KJ, MacNeil-Vroomen JL, Eslami S, Geerlings SE, de Rooij SE, Buurman BM. Transitional care interventions prevent hospital readmissions for adults with chronic illnesses. Health Aff. 2014;33(9): 1531-9.

8. Burke RE, Guo R, Prochazka AV, Misky GJ. Identifying keys to success in reducing readmissions using the ideal transitions in care framework. BMC Health Serv Res. 2014; 14:423.

9. Jack BW, Chetty VK, Anthony D, et al. A reengineered hospital discharge program to decrease rehospitalization: a randomized trial. Ann Intern Med. 2009; 150(3): 178-87.

10. Stauffer BD, Fullerton C, Fleming $\mathbf{N}$, et al. Effectiveness and cost of a transitional care program for heart failure: a prospective study with concurrent controls. Arch Intern Med. 2011;171(14):1238-43.

11. Voss R, Gardner R, Baier R, Butterfield K, Lehrman S, Gravenstein S. The care transitions intervention: translating from efficacy to effectiveness. Arch Intern Med. 2011;171(14):1232-7.

12. Centers for Medicare and Medicaid Services. Redmissions Reduction Program. https://www.cms.gov/medicare/medicare-fee-for-servicepayment/acuteinpatientpps/readmissions-reduction-program.html. Accessed 1/4/2016

13. Parry C, Mahoney E, Chalmers SA, Coleman EA. Assessing the quality of transitional care: further applications of the care transitions measure. Med Care. 2008;46(3):317-22.

14. Coleman EA, Parry C, Chalmers SA, Chugh A, Mahoney E. The central role of performance measurement in improving the quality of transitional care. Home Health Care Serv Q. 2007;26(4):93-104.

15. Elixhauser A, Steiner C, Harris DR, Coffey RM. Comorbidity measures for use with administrative data. Med Care. 1998;36(1):8-27.

16. The National Quality Forum. Specifications for the Three-Item Care Transition Measure - CTM-3. https://mhdo.maine.gov/_pdf/NQF_CTM_ 3_\%20Specs_FINAL.pdf. Accessed 1/4/2016

17. Hannan EL, Zhong $\mathbf{Y}$, Krumholz $\mathbf{H}$, et al. 30-day readmission for patients undergoing percutaneous coronary interventions in New York state. J Am Coll Cardiol Intv. 2011;4(12):1335-42.

18. Hannan EL, Zhong Y, Lahey SJ, et al. 30-day readmissions after coronary artery bypass graft surgery in New York state. J Am Coll Cardiol Intv. 2011;4(5):569-76.

19. Retrum JH, Boggs J, Hersh A, et al. Patient-identified factors related to heart failure readmissions. Circ Cardiovasc Qual Outcomes. 2013;6(2):171-7.

20. Englander H, Michaels L, Chan B, Kansagara D. The care transitions innovation (C-TraIn) for socioeconomically disadvantaged adults: results of a cluster randomized controlled trial. J Gen Intern Med. 2014;29(11):14607.

21. Medicare.gov Hospital Compare. Hospital Value-based Purchasing. http:// www.medicare.gov/HospitalCompare/linking-quality-to-payment.html. Accessed 1/4/2016. 
22. Amarasingham R, Moore BJ, Tabak YP, et al. An automated model to identify heart failure patients at risk for 30-day readmission or death using electronic medical record data. Med Care. 2010;48(11):981-8.

23. Hospital Consumer Assessment of Healthcare Providers and Systems. Summary of HCAHPS survey results: July 2013-June 2014, Discharges April 22, 2015

24. Mazor KM, Clauser BE, Field T, Yood RA, Gurwitz JH. A demonstration of the impact of response bias on the results of patient satisfaction surveys. Health Serv Res. 2002;37(5):1403-17.
25. Paganini-Hill A, Hsu G, Chao A, Ross RK. Comparison of early and late respondents to a postal health survey questionnaire. Epidemiology. 1993;4(4):375-9.

26. Gan SC, Beaver SK, Houck PM, MacLehose RF, Lawson HW, Chan L. Treatment of acute myocardial infarction and 30-day mortality among women and men. N Engl J Med. 2000;343(1):8-15.

27. Schulman KA, Berlin JA, Harless W, et al. The effect of race and sex on physicians' recommendations for cardiac catheterization. N Engl J Med. 1999;340(8):618-26. 